\title{
Uso de la tableta digital en entornos universitarios de aprendizaje a distancia
}

\section{Using the tablet in Distance University learning environment}

\author{
Martha Agila-Palacios \\ Universidad Técnica Particular de Loja, UTPL (Ecuador) \\ María Ramírez-Montoya \\ Instituto Tecnológico Superior de Monterrey, ITESM (México) \\ Ana García-Valcárcel \\ Universidad de Salamanca, USAL (España) \\ Jennifer Samaniego-Franco \\ Universidad Técnica Particular de Loja, UTPL (Ecuador)
}

\section{Resumen}

Los dispositivos móviles, entre ellos las tabletas digitales, se han convertido en una herramienta de trabajo en el ámbito académico, tanto en entornos presenciales como a distancia y/o virtuales; de ahí la necesidad de investigar cómo utilizan estos dispositivos los estudiantes, con qué frecuencia, cuáles son los tipos de interacción más comunes, si modifican en algo sus habilidades previas, estrategias en el uso de tecnologías, etc. La presente investigación se enfoca en la identificación de tipos de interacción de los estudiantes que utilizan la tableta digital como material de aprendizaje en modalidad a distancia con soporte en un Entorno Virtual de Aprendizaje (EVA) a través de técnicas de minería de datos (clusterización) siguiendo la metodología CRISP-DM. Los resultados obtenidos muestran que casi la totalidad de los estudiantes usan la tableta para la gestión de su material bibliográfico base y el $76 \%$ únicamente para la lectura de los mismos (interacción básica), solamente el 21\% la utilizan para otras actividades relacionadas con la comunicación (interacción comunicativa -social) y un 10\% para actividades orientadas a producir y compartir contenido (interacción productiva). Estos resultados no están directamente relacionados con la interacción en el EVA, pues no necesariamente aquellos que usan la tableta para comunicación y producción de contenidos son aquellos que tienen una interacción alta en el EVA.

Palabras clave: universidad a distancia; comportamiento del estudiante; aprendizaje móvil; estudiante universitario de primer ciclo; tableta digital. 


\begin{abstract}
Mobile devices, including tablets, have increasingly become a working tool in the academic field, both in face-to-face and distance and/or virtual environments; hence the need to investigate how often students use these devices, what for, what the most common types of interaction are, and if they modify their previous skills, and so forth. The present research focuses on the determination of types of interaction of students using the digital tablet as learning material in distance education with support in a Virtual Learning Environment (VLE) through data mining techniques (clustering) using the CRISP-DM methodology. The results show that almost all students use the tablet to manage their basic bibliographic material and $76 \%$ only use it to read their books (basic interaction), also only $21 \%$ of them use it for other activities related to communication (communicative and social interaction) and a $10 \%$ for activities oriented to producing and sharing content (productive interaction). These results are not directly related to the interaction in the VLE, since those students who use the tablet for communication and content production are not necessarily the ones that have high interaction in the VLE.
\end{abstract}

Keywords: distance learning university; student behaviour; m-learning; undergraduate student; tablet.

Con el pasar del tiempo los dispositivos móviles se han ido incorporando cada vez más a diferentes ámbitos de la vida de las personas. Diferentes organismos muestran estadísticas de los cambios acelerados en relación a los dispositivos móviles. Según el informe de Cisco VNI, para el 2017 habrá 5.200 millones de usuarios móviles, desde los 4.300 millones contabilizados en 2012 (Barnett, Sumits, Jain, y Andra, 2015). Sin embargo, según la Unión Internacional de Telecomunicaciones (ITU por sus siglas en inglés) de la ONU en su informe de "medición de la sociedad de la información del 2016, indica que en los países desarrollados el 95\% de las personas poseen un celular y, en los países en desarrollo, el 90\% (ITU, 2016). Esto nos da una pauta de los retos de investigaciones en diferentes ámbitos relacionados con la penetración de los dispositivos móviles.

Uno de los ámbitos de estudio de los dispositivos móviles es la educación, de ahí el término de un ambiente de aprendizaje denominado "aprendizaje móvil" o más conocido como m-learning o mobile learning en inglés. Según Traxler (2007) dar una definición única de m-learning es complicado, pues es personal, contextual y situado. Hay definiciones de m-learning que contemplan 3 componentes fundamentales: hardware, interface y diseño pedagógico (Sánchez, Olmos y GarcíaPeñalvo, 2013). Otras definiciones indican que m-learning es el uso de tecnología móvil en la educación (Ng, 2015) o, más específicamente, el uso de tecnología móvil para fomentar aprendizajes (Ramírez, 2012; Saleh y Bhat, 2015). En definitiva, m-learning es una modalidad o ambiente de aprendizaje que se da a través de dispositivos que permiten una movilidad tanto de contenido como de personas.

En este sentido, hay diversidad de investigaciones en torno al m-learning. Según Saleh y Bhat (2015), la mayoría de ellas se centra en la evaluación de estos 
sistemas de enseñanza-aprendizaje así como en su diseño. En cuanto al diseño, hay investigaciones respecto a los recursos educativos para dispositivos móviles, puntualmente los libros electrónicos y la lectura móvil (Nwagwu y Okafor, 2014; West y Ei, 2014), así como también a los dispositivos electrónicos que facilitan la lectura, puntualmente las tabletas (San Martín, Peirats y López, 2015). En relación con la evaluación de los sistemas m-learning, hay diferentes perspectivas y una de ellas es el análisis de las interacciones de los estudiantes, aspecto en el que aporta luz la presente investigación.

Cuando los estudiantes utilizan dispositivos móviles, ya sea para aspectos sociales o académicos, las interacciones generadas muestran patrones de comportamiento que dan pautas para la implementación de m-learning. López y Silva (2014) determinaron que el $75 \%$ de los estudiantes usan sus dispositivos móviles para actividades académicas, pese a que no todas las aplicaciones institucionales están diseñadas para móviles. Márquez de Oliveira (2012), identificaron patrones en cuanto a preferencia de soportes de lectura, un $40 \%$ se orientaron por los ebooks y un 60\% por los libros impresos. Así mismo, Shelburne (2013) estudió la aceptación de los ebooks en dos perfiles de estudiantes (pregrado y postgrado); en ambos casos su uso principal fue para la investigación. En esta investigación se muestran los diferentes tipos de interacciones de los estudiantes tanto con la tableta digital como en el entorno virtual de aprendizaje de forma independiente y cruzada.

\section{LAS TABLETAS DIGITALES COMO APOYO EN LA EDUCACIÓN SUPERIOR}

Uno de los dispositivos móviles utilizados actualmente como herramienta de trabajo en el proceso de enseñanza aprendizaje es la tableta digital y en torno a ella han surgido en los últimos años interesantes investigaciones. Así, San Martín, Peirats, y López (2015) determinaron, entre otros resultados, que al sustituir los libros de texto por tabletas digitales se incrementa la autogestión del proceso de aprendizaje. Rossing, Miller, Cecil y Stamper (2012) afirman que al incluir iPads en el aula se tienen beneficios tales como acceso aparentemente ilimitado a la información y ventajas para el aprendizaje colaborativo, aunque también tienen el potencial de distraer a los estudiantes y crear frustración en el aula. Así mismo, Navarro, Molina, Redondo y Juárez-Ramírez (2015), como resultado de su revisión sistemática de literatura, detectaron que la investigación en m-learning con tabletas digitales y smartphone, se ha incrementado significativamente desde 2013. Las investigaciones realizadas muestran una gran diversidad de contextos y variables de estudio.

Uno de los contextos, con particularidades en cuanto a perfil de estudiantes, tipos de recursos o en métodos y estrategias de enseñanza aprendizaje, es la educación superior. Dyson (2016), enfoca su investigación en la implementación de estrategias de aprendizaje activo en el aula con dispositivos móviles a fin de garantizar la sostenibilidad del m-learning. En cambio, Shelburne (2013) se enfoca a buscar 
actitudes y comportamientos de los estudiantes que utilizan libros electrónicos en una biblioteca de la Universidad de Illinois. En relación con las actitudes y comportamientos, Ramos, Herrera y Ramírez (2010) determinaron las habilidades cognitivas básicas (enfoque, búsqueda y recuperación de información, organización, análisis, evaluación y transformación) y habilidades cognitivas superiores (solución de problemas, toma de decisiones, pensamiento crítico, pensamiento creativo y "melioration") que se desarrollan al utilizar recursos m-learning en un contexto universitario. Así mismo, Organista-Sandoval, Serrano-Santoyo, Mc-anally-Salas y Lavigne (2013) indican que aunque la mayoría de los estudiantes universitarios tienen dispositivos móviles, pocos son aquellos que los utilizan para actividades académicas, entre ellas comunicación, manejo de información y organización; de ahí la importancia de suscitar el desarrollo de alternativas que promuevan el uso académico de los dispositivos móviles.

En relación a los dispositivos móviles, la tableta digital ha ido tomando importancia en los procesos de aprendizaje. Chen y Denoyelles (2013) indican que las tabletas emergen como poderosos dispositivos de aprendizaje porque son pequeñas y portátiles y el tamaño de la pantalla permite a los estudiantes recuperar y componer la información más fácilmente que los pequeños dispositivos móviles. Así mismo, Mang y Wardley (2012) encontraron que las tabletas representaban una distracción mucho menor para los estudiantes que las computadoras portátiles, pues eran menos propensos que los usuarios de computadoras portátiles a participar en actividades fuera de la tarea como mensajería instantánea, uso de redes sociales y ver videos durante una conferencia; por ello, la necesidad de explorar y explotar las características de las tabletas con estrategias que promuevan un compromiso académico tanto en estudiantes como en docentes.

\section{CONTEXTO DE LA INVESTIGACIÓN}

La presente investigación se llevó a cabo en la Universidad Técnica Particular de Loja (Ecuador) que ofrece estudios de grado y postgrado tanto en modalidad abierta y a distancia como presencial. Actualmente cuenta con alrededor de 31.000 estudiantes en modalidad abierta de grado y posgrado; y, con 6.500 estudiantes en modalidad presencial de grado y postgrado. En modalidad abierta y a distancia ofrece 16 titulaciones y en modalidad presencial 28 titulaciones.

El objetivo de la investigación se enfoca en estudiantes de primer ciclo de modalidad abierta y a distancia (MAD) en 83 centros universitarios de diferentes provincias del país, con una población de estudio de 8.034 estudiantes. Se eligió a los estudiantes de la carrera de "Derecho" por ser una de las más numerosas y cumplía con los criterios de selección de la muestra.

En el modelo educativo de la modalidad abierta y a distancia de la Universidad, el material bibliográfico básico de estudio es una guía didáctica y un texto básico de alguna editorial, los mismos que desde el periodo académico octubre 2015 - febrero 
2016, son entregados en formato digital, junto con el dispositivo de lectura (tableta digital) a todos los estudiantes de primer ciclo.

En cada tableta se dispone de un escritorio personalizado con acceso directo al gestor de material bibliográfico y a servicios principales de la Universidad, como por ejemplo: el Entorno Virtual de Aprendizaje, Biblioteca Virtual, entre otros. El gestor de material bibliográfico le permite visualizar los libros y guías de manera organizada por periodos académicos y por asignatura y acceder directamente al lector que es propio de la institución, lo que permite la recolección de las trazas de navegación por el libro.

\section{OBJETIVOS Y METODOLOGÍA}

La presente investigación tiene como objetivo determinar los perfiles de estudiantes que pueden surgir de acuerdo al uso de la tableta digital como recurso de aprendizaje en modalidad abierta y a distancia de la Universidad Técnica Particular de Loja (Ecuador), a fin de determinar un contexto base de implementación de un ambiente de aprendizaje m-learning.

Para el análisis de los datos se ejecutaron procesos de minería de datos a través de la metodología Crisp - DM (Cross Industry Standard Process for Data Mining), que según un estudio de Kdnuggets, en comparación con otras metodologías, se convirtió en la más utilizada en proyectos de minería de datos (Kdnuggets, 2014). Las fases son: comprensión del negocio, comprensión de los datos, preparación de los datos, modelado y evaluación.

En la comprensión del negocio se logró afianzar los objetivos del proyecto de minería, supuestos, riesgos, restricciones y los criterios de éxito. Entrelas restricciones algo importante que se determinó es la limitante de acceso a información de área de trabajo e ingresos económicos de los estudiantes, lo cual nos podía dar información adicional para determinar si estos valores influyen o no en la forma en que se usa la tableta digital; así mismo, no es posible obtener la información del comportamiento de los estudiantes en cada una de las aplicaciones que descarga, ya que no son propiedad de la institución.

En la fase de comprensión de los datos se definió el tipo de muestreo no probabilístico por criterios, estos son:

- Individuos que poseen tableta digital.

- Carrera/titulación con mayor número de estudiantes.

- Estudiantes de diferentes centros universitarios del país.

Para obtener el tamaño de la muestra, aplicando el primer criterio a la población total (8.034) se seleccionaron 7.196 estudiantes; de ellos, y como resultado de aplicar la fórmula para la muestra en poblaciones finitas con un nivel de confianza del 95\%, 
se obtuvo que el tamaño de la muestra es de 1.468, la misma que se la obtuvo de la carrera o titulación de Derecho que es la que posee mayor número de estudiantes.

Las bases de datos de donde se extrae la información de la muestra bajo los criterios antes mencionados, corresponden a los siguientes sistemas:

- Entorno Virtual de Aprendizaje: Plataforma donde se registra la interacción del estudiante con el contenido, actividades, compañeros y profesores; entre las actividades destacadas están el chat, foro y videocolaboración que son las que como mínimo deben estar presentes en cualquier aula virtual. Las variables a utilizar son:

Tabla 1. Campos de información del Entorno Virtual de Aprendizaje

\begin{tabular}{|l|l|l|}
\multicolumn{1}{|c|}{ Tabla } & \multicolumn{1}{c|}{ Campo } & \multicolumn{1}{c|}{ Descripción } \\
\hline mdl_user & User id & Código identificador del estudiante \\
\hline & Id number & Número de cédula del estudiante \\
\hline mdl_log & User id & Código identificador del estudiante \\
\hline & Module & Actividad \\
\hline & Action & Acción \\
\hline
\end{tabular}

Elaboración propia

- Sistema Académico Syllabus, donde se registra tanto la información personal al momento de la matrícula como los resultados de notas en cada una de las asignaturas. De este sistema se utilizaron las siguientes variables:

Tabla 2. Campos de información del sistema académico

\begin{tabular}{|l|l|}
\hline \multicolumn{1}{|c|}{ Campo } & \multicolumn{1}{c|}{ Descripción } \\
\hline identificación & Número de cédula del estudiante \\
\hline fecha_nacimiento & Fecha de nacimiento \\
\hline genero & Género \\
\hline estado_civil & Estado civil \\
\hline centro & Centro Universitario en el que está matriculado \\
\hline
\end{tabular}

Elaboración propia

- Sistema de gestión de material bibliográfico: en este sistema se registra toda la interacción del estudiante con la tableta digital. Las variables que se obtuvieron son: 
Tabla 3. Campos de información del Sistema de Gestión de material bibliográfico

\begin{tabular}{|l|l|l|}
\hline \multicolumn{1}{|c|}{ Vista } & \multicolumn{1}{c|}{ Campo } & \multicolumn{1}{c|}{ Descripción } \\
\hline \multirow{2}{*}{ bi_usuario } & id_usuario & Identificador del estudiante \\
\cline { 2 - 3 } Bidispositivo & Cedula & Número de cédula del estudiante \\
\hline \multirow{2}{*}{ bidispositivousuario } & id_dis_fisico & Número de identificación del dispositivo. \\
\cline { 2 - 3 } & id_dis_fisico & Número de identificación del dispositivo \\
\cline { 2 - 3 } & id_usuario & Identificador del estudiante \\
\hline \multirow{2}{*}{ ihistoricoaplicacion } & id_aplicacion & Identificador de la aplicación \\
\cline { 2 - 3 } & id_dis_fisico & Identificador del dispositivo \\
\hline \multirow{3}{*}{ Biaplicacion } & id & Número de identificación del dispositivo \\
\cline { 2 - 3 } & nombre & Nombre de las aplicaciones \\
\cline { 2 - 3 } & paquete & Nombre del paquete de la aplicación \\
\hline
\end{tabular}

Elaboración propia

Como resultado de esta fase se determinó que la mayoría de estudiantes tienen 18 años; en cuanto al estado civil, la mayoría son solteros con un 64\%; y en relación al género, la distribución es equilibrada, con un 54\% de hombres.

En la fase de preparación de los datos se analiza en cada variable, los datos que ofrecen información respecto al comportamiento de los estudiantes, datos erróneos o faltantes y se obtiene el conjunto de datos finales. En esta fase el mayor filtrado se realizó en los datos de las variables, module y action de la base de datos del EVA, ya que algunas de ellas estaban vacías y se enfocó en los módulos y acciones que mayormente han sido utilizadas. Así mismo, las variables estado civil y edad registraban valores inconsistentes, por lo que dichos registros fueron eliminados.

En la fase de modelado y por los objetivos planteados en la investigación se decidió usar la técnica de clusterización, tanto para la participación en el EVA como en la interacción con la tableta. Esta técnica tiene como objetivo la agrupación de datos que formen clústeres o conglomerados que sean homogéneos internamente y heterogéneos externamente. Previo a la implementación de esta técnica, es necesario realizar el análisis factorial para determinar si se requiere la reducción de variables para tener grupos homogéneos sin alterar información relevante.

En el caso de los datos de la tableta digital, puntualmente los nombres de las aplicaciones y juegos, antes de iniciar el análisis factorial en vista de la amplia diversidad de valores, fue necesario agruparlos manualmente por categorías teniendo los siguientes resultados en cuanto a distribución de frecuencias:

- Aplicaciones_comunicacion con un 54.9\%.

- Aplicaciones_lector con un 75.3\%.

- Aplicaciones_mediosVideos con un 55.9\%. 
- Aplicaciones_productividad con un 20.2\%.

- Aplicaciones_sociales con un 25.5\%.

En el caso de los juegos, el 82,7\% de estudiantes no utiliza juegos en la tableta, y el 17,3\% utilizan muy diferentes tipos de juegos, el más frecuente es utilizado por el 3,7\%, resultando un valor extremadamente bajo, lo que pone de manifiesto que se trata de una variable no homogénea dificultando su comparación.

Para tomar una decisión con respecto al análisis factorial se realizaron las siguientes pruebas:

- Matriz de correlación.- define el grado de relación entre variables y, dependiendo de su valor resultante (determinante), se decide si es pertinente ejecutar el análisis factorial. Mientras sea cercano a cero pero no necesariamente cero, es recomendable aplicar el análisis factorial.

- KMO (Kaiser-Meyer-Olkin).- es un índice que permite la comparación entre las magnitudes de los coeficientes de correlación observados y las magnitudes de los coeficientes de correlación parcial (Quintín y Paz, 2007).

- La prueba de esfericidad de Bartlett indica que si la correlación entre variables es menor a 0,05, es apropiado realizar el análisis factorial.

- Porcentaje de varianza.- este método se basa en "tomar como número de factores el número mínimo necesario para que el porcentaje acumulado de la varianza total explicada alcance un nivel satisfactorio $(75 \%, 80 \%)$ " (De la Fuente Fernández, 2011c, p.13).

Los resultados obtenidos en las cuatro pruebas (Ver tabla 4) con los datos de interacción del EVA y de la tableta digital muestran, en su mayoría, que no es necesario realizar el análisis factorial; por ello, se concluye que las variables no están correlacionadas entre sí y se procede a realizar el análisis de clusterización.

Tabla 4. Resultados de pruebas previas al análisis factorial

\begin{tabular}{|l|l|l|l|l|}
\hline \multirow{2}{*}{ Prueba } & \multicolumn{2}{c|}{ EVA } & \multicolumn{2}{c|}{ Tableta } \\
\cline { 2 - 6 } Matriz de correlación & Valor & Recomendable & \multicolumn{1}{c|}{ Valor } & Recomendable \\
\hline KMO & 0,736 & No & 0,290 & No \\
\hline Barlett & 0,653 & No & 0,563 & No \\
\hline Varianza & 0,000 & Si & 0,000 & Si \\
\hline
\end{tabular}

Elaboración propia 
A continuación se realiza la clusterización mediante la herramienta Weka y SPSS para los datos del EVA y SPSS para los datos de la tableta digital; con ellas se obtienen los clústeres de estudiantes a los que se los ha categorizado por nivel de participación y perfil según las actividades que realizan.

En el caso del EVA, con las dos herramientas se testeó con 2, 3 y 4 clústeres y según la homogeneidad de la distribución en los clústeres, se elige trabajar con 3. La única diferencia es que en SPP permite visualizar el clúster asignado a cada uno de los estudiantes y es por ello que también se trabajó con esta para los datos de la tableta, a fin de poder establecer una relación de la interacción en las tabletas y en el EVA.

\section{RESULTADOS}

\section{Interacción en el EVA}

Según los clúster obtenidos, se clasifica a los estudiantes en las siguientes categorías:

- Interacción baja.- son estudiantes que mayoritariamente no tienen una participación en el chat, foro y mensajes aunque en la vista de recursos sí presentan una interactividad mayor a cero. Representan el 56\% de los estudiantes.

- Interacción media.- su característica es la de interacción moderada, aspecto definitorio por la vista de recursos, ya que en las actividades de chat, postear en el foro y escribir mensajes la diferencia es mínima. Se incluyen en este grupo el $35 \%$ de los estudiantes.

- Interacción alta.- son estudiantes que presentan una interacción mayor en cuanto a vista de recursos y en el chat con un mínimo de diferencia en relación a los otras categorías. Está constituido por el 9\% de los estudiantes. 
Figura 1. Interacciones en el EVA

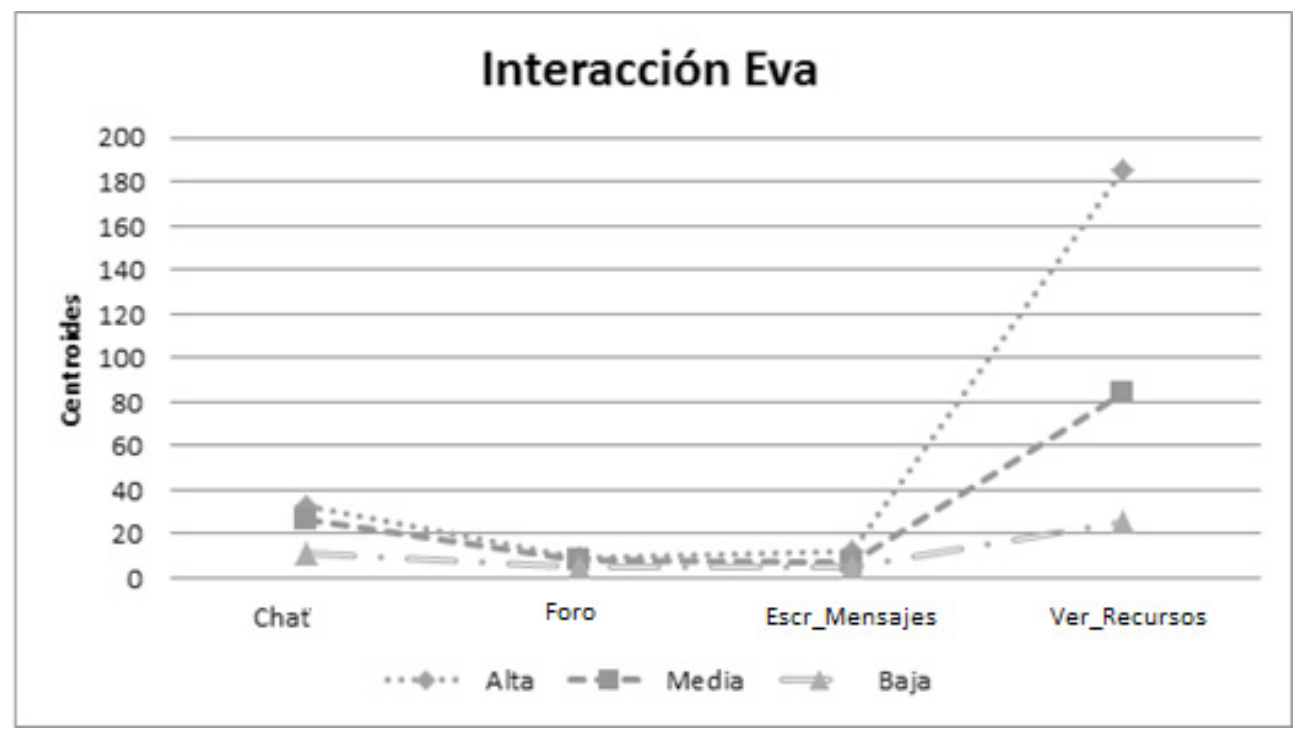

Elaboración propia

Como se observa en la figura 1, la variable que determina la diferenciación de los grupos es fundamentalmente la visualización de recursos $\mathrm{y}$, en menor medida, la participación en el chat. En relación a la participación en foros y envío de mensajes los 3 grupos tienen valores muy bajos y similares entre sí.

Dentro de estos grupos, en relación a la edad, el estado civil y el género, se obtuvieron los siguientes resultados:

- Del 9\% de estudiantes que tienen una interacción alta, la mayoría son varones con estado civil soltero y su edad fluctúa entre 28 y 33 años.

- Del 35\% de estudiantes que tienen una interacción media, el mayor porcentaje son mujeres con estado civil soltero entre 22 a 27 años.

- Finalmente, del 56\% de estudiantes que tienen una interacción baja, la mayoría son hombres con estado civil soltero entre 16 a 21 años.

\section{Interacción en la Tableta Digital}

A diferencia de la interacción en el EVA, aquí se encontró una característica general, pues la mayoría de estudiantes $(99,9 \%)$ usan la tableta para acceder a la plataforma y descargar $e$-books mediante el aplicativo del escritorio UTPL y en un $75,3 \%$ para la lectura de sus $e$-books. 
Con base en las diferencias de esta población, en cuanto a interacción con otras aplicaciones, se establecieron las siguientes categorías:

- Interacción básica: estudiantes que adicional a la descarga, lectura del e-books y acceso al EVA, utilizan muy poco o nada otro tipo de aplicaciones. Este grupo representa al 69\% de la muestra.

- Interacción comunicativa - social: este grupo tiene el nivel más alto de uso de aplicaciones de comunicación como: correo electrónico, navegadores, Skype, line, redes sociales, entre otros, y un uso moderado en cuanto a aplicaciones de videos como: youtube, vlc, entre otros. Representa el 21\% de la muestra.

- Interacción productiva: este grupo se diferencia de los demás ya que tiene el nivel más alto de uso de aplicaciones para producir y compartir contenido como: Dropbox, herramientas de ofimática, Prezzi, Google Drive, aunque también se caracteriza por usar aplicaciones de comunicación, sociales, medios y videos en menor medida que el grupo con interacción comunicativa. Representa el 10\% de la muestra.

Figura 2.Tipos de interacción en la tableta digital

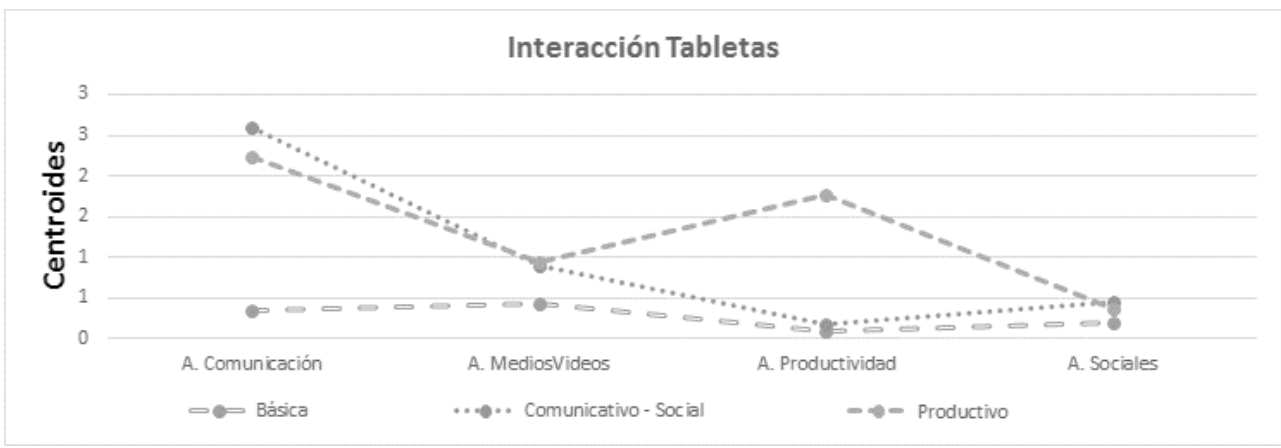

Elaboración propia

Dentro de estos grupos, en relación a la edad, el estado civil y el género, se obtuvieron los siguientes resultados:

- Del 69\% de estudiantes que tienen una interacción básica, la mayoría son hombres solteros entre 22 a 27 años.

- Del $21 \%$ de estudiantes que se caracterizan por tener una interacción comunicativo-social, la mayoría son mujeres solteras entre 16 a 21 años.

- Del 10\% de estudiantes con interacción productiva, en su mayoría son mujeres solteras entre los 22 a 27 años. 


\section{Relación entre la interacción en el EVA y en la tableta digital}

Para analizar la relación entre las interacciones que tienen los estudiantes en la tableta digital y en el EVA, se realizó el cruce de datos, obteniendo los siguientes resultados:

Tabla 5. Interacción Tableta Digital -EVA

\begin{tabular}{|l|l|c|c|c|c|}
\hline \multirow{2}{*}{ Interacción Tableta digital } & \multicolumn{3}{|c|}{ Interacción EVA } & \multirow{2}{*}{ Total } \\
\cline { 2 - 5 } & Alto & Medio & Bajo & \\
\hline Productivo & $1 \%$ & $4 \%$ & $5 \%$ & $10 \%$ \\
\hline Comunicativo-social & $3 \%$ & $8 \%$ & $10 \%$ & $21 \%$ \\
\hline Básica & $5 \%$ & $23 \%$ & $41 \%$ & $69 \%$ \\
\hline Total & $\mathbf{9 \%}$ & $\mathbf{3 5 \%}$ & $\mathbf{5 6 \%}$ & $\mathbf{1 0 0 \%}$ \\
\hline
\end{tabular}

Elaboración propia

Estos resultados muestran que:

- Del 56\% de estudiantes que tienen una interacción baja en el EVA:

- La mayoría de estudiantes, de igual manera tienen un patrón de interacción básica en la tableta digital, es decir, la usan solo para descargar los libros y leerlos.

- Un 10\% de ellos posee un patrón comunicativo - social.

- Un $5 \%$ presenta un patrón de productividad.

- Del 35\% de estudiantes que tienen una interacción media en el EVA:

- La mayoría de ellos tiene una interacción básica en la tableta.

- $\quad$ El 8\% son comunicativos - sociales.

- Solo un $4 \%$ son productivos.

- Del 9\% que tienen una interacción alta en el EVA:

- La mayoría de los estudiantes usan la tableta en forma básica y tan solo un $3 \%$ son comunicativos - sociales y un $1 \%$ productivos.

Para validar la robustez del modelo resultante se utilizó la prueba de verosimilitud, la de bondad de ajuste y la de R-cuadrado, dando resultados positivos en relación a sí mismo; mientras que, cuando se analizó la relación entre la interacción en el EVA y la interacción en la tableta digital, los resultados muestran que no hay una relación de dependencia, pues el R-cuadrado obtenido es 0,20. 
M. Agila-Palacios; M. Ramirez-Montoya; A. García-Valcárcel; J. Samaniego-Franco

Uso DE LA TABLETA DIGITAL EN ENTORNOS UNIVERSTTARIOS DE APRENDIZAJE A DISTANCIA

\section{DISCUSIÓN Y CONCLUSIONES}

Al igual que en otros países de Latinoamérica, en Ecuador se observa un alto porcentaje de hogares con un teléfono celular. Según el Observatorio TIC del Ministerio de Telecomunicaciones del Ecuador (2016), el 90,1\% de los hogares tiene al menos un teléfono celular en comparación con las computadoras de escritorio y laptop, que solo tienen el 26,7\% y 27,6\% de los hogares. Este porcentaje de posesión de teléfono celular está en relación con el informe de la UNESCO, que en el 2015 indica que de los 7 mil millones de habitantes, 6 mil millones tienen acceso a un teléfono celular, es decir, aproximadamente el 86\% (West y Ei, 2015). Esto representa una situación (gran cantidad de población con dispositivos móviles) que puede ser aprovechada para la educación, aunque no necesariamente sean teléfonos inteligentes o tabletas digitales.

Cuando hablamos de teléfonos inteligentes el porcentaje disminuye, pero más aún cuando nos enfocamos a las tabletas digitales. En Ecuador, según el observatorio TIC del Ministerio de Telecomunicaciones, hasta el 2015 solo el 1,92\% de los hogares usan la tableta para conectarse a Internet y el 8,43\% el celular. Sin embargo, el crecimiento proyectado es elevado, pues según el informe de Cisco VNI, en el 2015 el número de tabletas conectadas aumentó 1,3 veces y cada tableta generó 2,8 veces más tráfico que el teléfono inteligente promedio; para el año 2020 las tabletas digitales generarán casi ocho veces más tráfico que el generado en 2015 (Barnett, Sumits, Jain, y Andra, 2015). De ahí que la movilidad y ubicuidad que promueven y promoverán los dispositivos móviles en cualquiera que sea su versión (smartphone, tableta digital, phablets, etc.) no deja de lado ningún lugar del mundo, aunque sus incrementos y velocidades de penetración sean diferentes en cada región.

En este caso puntual de estudio, los estudiantes, pese a tener un dispositivo móvil, hacen poco uso de él para otras actividades académicas salvo la lectura. Solamente la cuarta parte de los estudiantes utilizan herramientas de comunicación y muy pocos utilizan aplicaciones que facilitan la producción y edición de contenidos. En relación a estos resultados, Organista-Sandoval, Serrano-Santoyo, Mc-anally-Salas y Lavigne (2013) determinaron que el uso educativo del celular es mínimo y, por ello, es necesario ampliar las investigaciones para su adecuada integración al proceso educativo. De ahí la imperiosa necesidad de que en el diseño de las estrategias de enseñanza-aprendizaje los docentes promuevan el uso de los dispositivos móviles como instrumentos o herramientas de aprendizaje.

No existe una relación directa entre la interacción en la tableta digital y la interacción en el entorno virtual de aprendizaje, es decir, no necesariamente aquellos estudiantes que tienen interacción alta en el EVA son aquellos que utilizan la tableta para otras actividades académicas aparte de la lectura. El índice de relación obtenido es bajo, lo que indica que no hay una relación directa entre interacción en el EVA y en la tableta. López y Silva (2014) encontraron que del 25\% de estudiantes que usan los dispositivos móviles para ingresar al Entorno Virtual de Aprendizaje, el 75\% 
los usa para fines académicos. Ello da lugar a profundizar en la investigación para determinar las razones de interacción en un sistema y otro, que podrían depender de las competencias digitales de los estudiantes, o de la facilidad de visualización de los recursos, o de la estrategia de enseñanza aplicada, entre otros.

Un patrón común que se encontró en la investigación es que casi la totalidad de estudiantes usan la tableta digital para la gestión de su material bibliográfico y lectura. Casi la totalidad de los estudiantes usan la tableta para acceder a la plataforma y descargar $e$-books mediante el aplicativo del escritorio UTPL y la gran mayoría para la lectura de sus libros. Esto confirma lo mencionado por San Martín, Peirats, y López (2015) cuando indican que, entre otros resultados, al sustituir los libros de texto por tabletas digitales, se incrementa la autogestión del proceso de aprendizaje. Este contexto puede ser explotado para ir más allá de la lectura de los libros base de cada asignatura y promover en los estudiantes el desarrollo de competencias enfocadas a la búsqueda y selección de información, así como también a la gestión de sus recursos.

\section{Reconocimientos}

Este trabajo de investigación se ha realizado dentro del Programa de Doctorado en II Formación en la Sociedad del Conocimiento de la Universidad de Salamanca. Se agradece también a la Universidad Técnica Particular de Loja, Ecuador por la beca otorgada para estudiar en este programa, así como también por acceso al contexto de investigación.

\section{REFERENCIAS BIBLIOGRÁFICAS}

Barnett, T. J., Sumits, A., Jain, S., y Andra, U. (2015). Cisco Visual Networking Index (VNI) Update Global Mobile Data Traffic Forecast. Vni, 2015-2020.

Chen, B., y Denoyelles, A. (2013). Exploring Students' Mobile Learning Practices in Higher Education. Educause Review Online, 1-11.

Dyson, L. E. (2016). Achieving sustainable mobile learning through studentowned devices and student-generated multimedia content. In W. Ng y $\mathrm{T}$. Cumming (Eds.), Sustaining mobile learning: Theory, Research and Practice (212-225). Routledge, UK.

ITU-International Telecommunication Union. (2016). Measuring the
Information Society Report. Recuperado de http://www.itu.int/en/ITU-D/ Statistics/Documents/publications/ misr2016/MISR2016-w4.pdf

Kdnuggets. (2014). What main methodology are you using for your analytics, data mining, or data science projects? Poll. Recuperado de http://www.kdnuggets. com/polls/2014/analytics-data-miningdata-science-methodology.html

López, F. A., y Silva, M. M. (2014). Patrones de m-learning en el aula virtual. Revista de Universidad y Sociedad del Conocimiento (RUSC), 11(1), 208-221. doi: https://doi. org/10.7238/rusc.v11i1.1902

Marques de Oliveira, S. (2012). E-textbooks usage by students at Andrews University: 
A study of attitudes, perceptions, and behaviors. Library Management, 33(8/9), 536-560.

Mang, C. F., y Wardley, L. J. (2012). Effective Adoption of Tablets in Post-Secondary Education: Recommendations Based on a Trial of iPads in University Classes. Journal of Information Technology Education: Innovations in Practice, 11, 17. Recuperado de http://jite.org/ documents/Vol11/JITEv11IIPp301317Mang1138.pdf

Navarro, C. X., Molina, A. I., Redondo, M. A., y Juárez-Ramírez, R. (2015). Framework para Evaluar Sistemas M-learning: Un Enfoque Tecnológico y Pedagógico. VaepRita, 3, 38-45. Recuperado de: http:// repositorio.grial.eu/handle/grial/399

Nwagwu, W. E., y Okafor, J.-L. (2014). Diffusion of ebooks among postgraduate students of the University of Ibadan, Nigeria. Library Review, 63(1), 86-109. doi: http://doi.org/10.1108/LR-04-2013$\underline{0056}$

Ng, W., y Cumming, T. M. (Eds.). (2015). Sustaining Mobile Learning: Theory, Research and Practice. Routledge, UK.

Observatorio tic del Ministerio de Telecomunicaciones del Ecuador (2015). Indicadores y estadística. Recuperado de http://www.observatoriotic.mintel.gob. ec/estadistica/

Organista-Sandoval, J., Serrano-Santoyo, A., McAnally-Salas, L., y Lavigne, G. (2013). Apropiación y usos educativos del celular por estudiantes y docentes universitarios. Revista Electrónica de Investigación Educativa, 15(3), 138-156. Recuperado de http://redie.uabc.mx/vol15no3/ contenido-organistaetal.html

Ramos, a. I., Herrera, J. a., y Ramírez, M. S. (2010). Desarrollo de habilidades cognitivas con aprendizaje móvil: Un estudio de casos. Comunicar, 17, 201-209. doi: https://doi.org/10.3916/C34-201003-20

Ramírez, M. S. (2012). Métodos y estrategias de enseñanza para ambientes innovadores. Monterrey, México: Editorial digital del Tecnológico de Monterrey.

Rossing, J. P., Miller, W. M., Cecil, A. K., y Stamper, S. E. (2012). iLearning: The future of higher education? Student perceptions on learning with mobile tablets. Journal of the Scholarship of Teaching and Learning, 12(2), 1-26. Recuperado de http://josotl.indiana.edu/ article/view/2023

Sanchez Sanchez, J. C., Olmos Migueláñez, S., y García-Peñalvo, F. J. (2013). Mobile learning: Tendencies and lines of research. ACM International Conference Proceeding Series, 473-480. doi: http:// doi.org/10.1145/2536536.2536609

San Martín, Á., Peirats, J., y López, M. (2015). Las tabletas y la gestión de los contenidos digitales en los centros escolares. Revista Iberoamericana de Educación, 67, 139158.

Saleh, S. A., y Bhat, S. A. (2015). Mobile Learning: A Systematic Review. International Journal of Computer Applications, 114(11), 1-5.

Shelburne, W. A. (2013). E-book usage in an academic library: User attitudes and behaviors. Library Collections, Acquisitions, \& Technical Services, 33(23), 59-72.

Traxler, J. (2007). Defining, discussing, and evaluating mobile learning: The moving finger writes and having writ... International Review of Research in Open and Distance Learning, 8(2).

West, M., y Ei, H. C. (2014). Reading in the mobile era. Paris: Unesco Recuperado de http://unesdoc.unesco.org/ images/o022/002274/227436e.pdf 


\section{PERFIL ACADÉMICO Y PROFESIONAL DE LAS AUTORAS}

Martha Agila-Palacios. Ingeniera en Sistemas Informáticos, Magister en Educación a Distancia (UTPL- Ecuador). Cursando doctorado en Formación en la Sociedad del Conocimiento de la Universidad de Salamanca (España). Docente bimodal y directora de Tecnologías para la Educación del Vicerrectorado de MADUTPL. Líneas de investigación: e-learning, m-learning., competencias digitales, aprendizaje activo.

E-mail: mvagila@utpl.edu.ec

\section{DIRECCIÓN DE LA AUTORA}

Departamento de Ciencias de la Computación y electrónica

Universidad Técnica Particular de Loja

San Cayetano Alto, Marcelino Champagnat

Loja (Ecuador)

María Ramírez-Montoya. Profesora investigadora titular de la Escuela de Educación, Humanidades y Ciencias Sociales del ITESM (México) en los programas de maestría y doctorado de educación. Es directora de la Cátedra UNESCO: "Movimiento educativo abierto para América Latina", directora de la oficina del International Council for Open of Distance Education (ICDE): OER Latin America y coordinadora del grupo de investigación de "Innovación de Modelos Educativos" del ITESM. Es doctora en Filosofía y Ciencias de la Educación por la Universidad de Salamanca. Líneas de investigación: estrategias de enseñanza, recursos tecnológicos para la educación, formación de investigadores educativos y movimiento educativo abierto.

E-mail: solramirez@itesm.mx

\section{DIRECCIÓN DE LA AUTORA}

Instituto Tecnológico Superior de Monterrey Av. Eugenio Garza Sada 2501 Sur, Tecnológico, 64849

Monterrey, N. L. (México)

Ana García-Valcárcel. Licenciada y Doctora en Ciencias de la Educación por la Universidad de Comillas (Madrid). Catedrática de Tecnología Educativa (Área de Didáctica y Organización Escolar) en la Facultad de Educación de la Universidad de Salamanca. Departamento de Didáctica, Organización y Métodos de Investigación. Co-directora del Grupo de Investigación de Excelencia GITE-USAL: http://gite213. usal.es

E-mail:anagv@usal.es 


\section{DIRECCIÓN DE LA AUTORA}

Facultad de Educación.

Universidad de Salamanca

Paseo de Canalejas, 169. 37008

Salamanca (España)

Jennifer Samaniego-Franco. Ingeniera en Sistemas Informáticos y Computación. Trabaja como analista de innovación educativa para la Universidad Técnica Particular de Loja. Sus líneas de investigación y proyectos se centran en e-Learning, comunidades virtuales, perfiles de aprendizaje, tecnologías educativas y minería de datos.

E-mail: jbsamaniego@utpl.edu.ec

\section{DIRECCIÓN DE LA AUTORA}

Dirección de Tecnologías para la Educación

Universidad Técnica Particular de Loja

San Cayetano Alto, Marcelino Champagnat

Loja (Ecuador)

Fecha de recepción del artículo: 15/12/2016

Fecha de aceptación del artículo: 24/02/2017

\section{Como citar este artículo:}

Agila-Palacios, M., Ramírez-Montoya, M., García-Valcárcel, A., y SamaniegoFranco, J. (2017). Uso de la tableta digital en entornos universitarios de aprendizaje a distancia. RIED. Revista Iberoamericana de Educación a Distancia, 2O(2), pp. 255-271. doi: http://dx.doi.org/10.5944/ried.20.2.17712 